IASSNS $93 / 62$

TALPP $2106-93$

\title{
GAUGE AND GROUP PROPERTIES OF MASSLESS FIELDS IN ANY DIMENSION
}

\author{
N. Shnerb \\ Department of Physics, Bar-Ilan University \\ Ramat-Gan 52900, Israel \\ and \\ L.P. Horwitz $\S$ \\ School of Natural Sciences \\ Institute for Advanced Study \\ Princeton, N.J. 08540
}

PACS : 03. $10 .+\mathrm{k}$. 11.30.cp, 04.30.+x, 11.10.Kk .

\begin{abstract}
It is shown that the solvable structure of the stability group of a massless field yields, in a simple and short way; useful information about the physical polarization states, and the structure of the Hilbert space for such a field. It is also shown that such fields are necessarily gauge fields: the exact form of the gauge transformation follows from the structure of the equivalence classes implied by the group

$\S$ Permanent address: School of Phỵsics. Raymond and Beverly Sackler Faculty of Exact Sciences. Tel-Arir Liniversity, Ramat-Arir, Israel; also at Department of Phỹsics. Bar-Ilan Linirersity: Ramat-Gan , Israel
\end{abstract}


properties. Some examples for spinor and tensor fields in general dimensions are worked out and the structure of the Hilbert space for gravitational radiation is discussed in some detail. 


\section{Introduction}

The relations between the physical properties of the four dimensional massless fields and the representation of the Poincaré group have been treated by many authors [1-3]. It appears that in almost all cases in which this subject is treated, it is assumed that the field has a definite structure, e.g., that the massless vector field is a gauge field. Weinberg [4] has, howerer, treated this problem without assuming that the massless fields are gauge fields, and without specific assumptions on the form of the equations of motion. He starts by defining tensor fields transforming according to the $(0, j)$ or $(j .0)$ representations of the homogeneous Lorentz group (corresponding, for $j=1$ and 2 . to the Maxwell field strengths $F^{\mu \nu}$ and the Riemann - Christofel tensor $R^{\mu \nu \lambda \sigma}$ ). He shows that any corariant free field can be constructed as a linear combination of these fields and their derivatives. They cannot, howerer, be used to construct the interaction Hamiltonian. since the coefficients of the annihilation and creation operators for particles of momentum $p$ and spin $j$ ranish as $p^{j}$ for $p \rightarrow 0$. inconsistent with the existence of long range force laws. One must therefore use potentials. It is then explicitly shown that the vector potentials do not transform as tensors under Lorentz transformation. A Lorentz transformation induces a tensor transformation and additional terms which are a derivatives of a scalar function of $x, \Lambda$; these additional terms are understood as a gauge transformation, since they leave the field strength invariant. The only interactions allowed are therefore those which satisfy gauge invariance, i.e., they must be coupled to conserved currents. One finds, moreorer. that a non 
covariant term must be added to the interaction Hamiltonian (e.g., the Coulomb interaction for $j=1$ ) to obtain an invariant S-matrix [5]

In this paper, we obtain Weinberg's results from a different point of riew. This procedure providing some additional insight into the basic underlying mathematical structure. It follows directly from its properties as a (massless) representation of the Poincaré group that the massless potential field in any dimension must be a field with gauge degrees of freedom. Moreover. we show that the existence and the form of the gauge follows from the requirement that the field, as a representation of a group which has a solvable factor, must hare an equivalence class that corresponds exactly to the property of gauge invariance. This new theorem enables us to analyze the gauge properties of a massless field in any dimension, yielding the explicit form of the gauge and also (without use of the wave equation) the structure of the Hilbert space for the second quantized field theory. As examples for the use of our theorem, we investigate the problem of massless tensor and spinor fields for several cases, and discuss, in particular.the gauge freedom of the massless vector field in any dimension and the possibility of a gravitational, gauge invariant conformal field for $d=6$. For a spinor field, we find that there are two possibilities, i.e., spinor fields may be gauge fields, or may have a restricted handedness and then violate parity. We furthermore prove that the harmonic coordinate condition for the linearized gravitational field theory, which appears in the standard literature as a consequence of the freedom to make a general coordinate transformation, in fact, follows, as a condition for the existence of a massless spin-2 tensor field in flat space-time. 


\section{Vector Fields in General Dinension}

Let $A(x)$ be some field in an arbitrary flat manifold, a generalized spacetime, with metric $g$ of signature $(p, q)$ and suppose that it has some definite transformation properties, i.e., as a spinor or tensor field. Let us consider the Fourier transform $A(k)$. We define $A(k)$ as a massless field if the field has support for $k$ such that $k^{\alpha} k_{\alpha}=0$. Let $\Lambda_{\beta}^{\alpha}$ be an element of the homogeneous matrix-valued isometry group $A$ of the manifold. and clenote by $\tilde{\Lambda}_{\beta}^{\alpha}$ an element of the subgroup of $\tilde{I}(k)$ that stabilizes $k^{a}$, i.e.

$$
\tilde{\Lambda}_{3}^{a} k^{3}=k^{3} .
$$

where $\tilde{I}(k)$ is the little group (or stability group) of $k$. Under the action of the little group.

$$
A^{\prime}\left(k^{\prime}\right)=A^{\prime}(j k)=A^{\prime}(k)
$$

If $A$ is a scalar field, then it is invariant under this subgroup of transformations, but if $A$ has indices with spinor or tensor transformation properties then $A^{\prime} \neq A$. We shall define the components which span an irreducible representation of the (universal covering group of the) little group $\bar{\Lambda}(k)$ as the polarizations of the field. They are the physical spacetime degrees of freedom of the field. In the case of massless fields, one can show that the little group is a semidirect product of a maximally connected semisimple subgroup and a "translation" part which is a maximally simply connected solvable normal subgroup. There is a general theorem that in such a case the "translation" subgroup must be represented trivially in any finite dimensional irreducible representation of the group [6]. In this paper we use 
this property to find useful information about the field in a simple and general way:

In the four dimensional Minkowshi manifold, the little group of a massless field is isomorphic to $E(2)$, the isometry group of the Euclidian plane (for example, [6]). This group, for example, for $k$ in the $\tilde{z}$ direction, has 3 generators of the form [4]

$$
J_{3}=i\left(\begin{array}{cccc}
0 & 0 & 0 & 0 \\
0 & 0 & 1 & 0 \\
0 & -1 & 0 & 0 \\
0 & 0 & 0 & 0
\end{array}\right) \quad I_{1}=i\left(\begin{array}{cccc}
0 & 1 & 0 & 0 \\
1 & 0 & 0 & -1 \\
0 & 0 & 0 & 0 \\
0 & 1 & 0 & 0
\end{array}\right) \quad L_{2}=i\left(\begin{array}{cccc}
0 & 0 & 1 & 0 \\
0 & 0 & 0 & 0 \\
1 & 0 & 0 & -1 \\
0 & 0 & 1 & 0
\end{array}\right)
$$

and the algebra is

$$
\left[J_{3} \cdot L_{1}\right]=i L_{2}, \quad\left[J_{3}, L_{2}\right]=-i I_{1}, \quad\left[L_{1}, L_{2}\right]=0
$$

$L_{1}$ and $L_{2}$ generate the "translation" subgroup. Here the group is solvable and admits only one-dimensional (or infinite dimensional) representations so that $t^{\mu}$ (finite dimensional) must be stable under the action of the translation part. Since the translation generators are nilpotent, the finite translations are represented as:

$$
\begin{aligned}
& T_{1}(\alpha)=e^{-i \alpha L_{1}}=\left(\begin{array}{cccc}
\frac{1+\alpha^{2}}{2} & \alpha & 0 & \frac{-\alpha^{2}}{2} \\
\alpha & 0 & 0 & -\alpha \\
0 & 0 & 1 & 0 \\
\frac{\alpha^{2}}{2} & \alpha & 0 & \frac{1-\alpha^{2}}{2}
\end{array}\right) \\
& T_{2}(\beta)=e^{-i \beta L_{2}}=\left(\begin{array}{cccc}
\frac{1+3^{2}}{2} & 0 & \beta & \frac{-\beta^{2}}{2} \\
0 & 0 & 1 & 0 \\
\beta & 0 & 0 & -\beta \\
\frac{\beta^{2}}{2} & 0 & \beta & \frac{1-\beta^{2}}{2}
\end{array}\right)
\end{aligned}
$$

The field $-^{\mu}$ transforms under the translational part as

$$
A^{\prime \prime}(k)=\left(\begin{array}{c}
A^{t}+\eta \Delta+\alpha A^{x}+\beta \cdot A^{y} \\
A^{x}+\alpha \Delta \\
A^{y}+\beta \Delta \\
A^{2}=+\eta \Delta+a \cdot A^{x}+\beta \cdot A^{y}
\end{array}\right)
$$


where $\eta \equiv \frac{\alpha^{2}+3^{2}}{2}$ and $\Delta \equiv A^{t}-A^{*}$. If $A^{\mu}$ is required to be literally stable under this transformation, one finds that $\Delta=A^{x}=A^{y}=0$. Under the assumption of covariant commutation relations for the creation and annihilation operators,

$$
\left[a^{\mu}(k), a_{\nu}^{\dagger}\left(k^{\prime}\right)\right]=\delta^{\mu}{ }_{\nu} \delta\left(k-k^{\prime}\right)
$$

we find that this field is associated only with zero norm states. Such a field could exist classically but not quantum mechanically. In order to obtain a nontrivial field which satisfies these conditions, we must admit an equivalence relation in (part of the) rector field. i.e., to assume that $A^{j}$ is equivalent to $A^{j}$ plus an additive term of a given type. If we attempt to find a solution with $\Delta \neq 0$ we find that all the field components are defined only up to an equitalence relation. and the theorg again loses its physical meaning. One must then take $\Delta=0$. but $A^{x},-A^{y} \neq 0$ (one can not take just $A^{x}$ or just $A^{y}$ as physical fields because $J_{3}$ mixes them). We find that:

$$
\begin{aligned}
& A^{t}(k) \sim A^{t}(k)+\alpha A^{x}(k)+\beta A^{y}(k) \\
& A^{z}(k) \sim A^{z}(k)+\alpha \cdot A^{x}(k)+\beta \cdot A^{y}(k)
\end{aligned}
$$

This freedom corresponds to the gauge transformation:

$$
A^{\mu}(k) \longrightarrow A^{\mu}(k)+k^{\mu} \Lambda(k)
$$

where we identify $\Lambda(k)$ as $\alpha A^{x}(k)+\beta A^{y}(k)$. The Fourier transform of (1I) is:

$$
\begin{gathered}
A^{\mu}(x) \longrightarrow A^{\mu}(x)+\partial^{\mu} \Lambda(x) \\
-7-
\end{gathered}
$$


which is the well known gauge freedom of the four dimensional electromagnetic field. We conclude that the existence of non-trivial physical degrees of freedom for a massless vector field in $3+1$ dimensions implies that the field must be a gauge field. Moreover, one identifies in this way the nature of the gauge group and the form in which these physical degrees of freedom are represented by the field.

The structure of the quantum mechanical Hilbert space is determined by the physical degrees of freedom (i.e., the polarization states); we discuss this structure in the following.

The conditions $\Delta=0$ or $A^{t}=A^{\text {z }}$ cannot be satisfied (quantum mechanically) as an operator iclentity [ [] ; instead, one imposes the subsidiary condition by restricting the space of the states by some linear condition, such as the GuptaBleuler condition:

$$
\left(a^{t}-a^{z}\right)|\nu\rangle=0
$$

for any physical state $|\nu\rangle$. The space of the states, therefore, contains a physical, positive definite norm subspace $\left.\left\{\mid \nu_{p h y s}\right)\right\}$ created by $a^{\dagger^{\dagger} x}$ and $a^{\dagger y}$ (or by $\left(\frac{1}{\sqrt{2}}\right)\left(a^{\dagger x} \pm\right.$ $\left.a^{\dagger y}\right)$, which creates \pm 1 helicity eigenstates), which we call the physical Hilbert space, and a "ghost" sector that contain at least 1 "ghost" created by $a^{\dagger^{\dagger}}+a^{\dagger}$. The ghost sector is a zero norm subspace orthogonal to the physical one. The physical Hilbert space can be defined covariantly as the closure of the quotient space $\left\{\left|\nu_{p h y s}\right\rangle\right\} /\left\{\mid \nu_{\text {ghost }}\right\}$ and the $S$ matrix is unitary with respect to this physical subspace if it is norm-preserving in the whole Hilbert space and the subspace $\left|\nu_{p h y s}\right\rangle$ is invariant under its action [7].

In general dimension $d$ with signature $(p . q)$, it is easy to see that the little 
group of the massless field with null $k$ is $E(p-1, q-1)$, the Euclidian isometr: group of the $d-2$ dimensional space with signature (p-1,q-1). Let us take the zero length vector in a form in which there is just one timelike and one spacelike component, which we shall call longitudinal components. In the construction of the matrices of the stability subgroup, one sees that in addition to the $O(p-1, q-1)$ part, there are elements that connect the non-zero components of the zero length vector. These form a commuting set of $d-2$ boosts and rotation matrices on the $(p, q)$ space which play the role of the "translations" of $E(p-1 . q-1)$. The semisimple part of this group is $O(p-1, q-1)$ and the maximally solvable subgroup is $T(d-2)$ where $T$ is the "translation" part (one can see here in general the same structure as for the example of $O(3,1))$.

In a general representation of the massless vector $k$, we denote by $A^{\text {th }}$ the "longitudinal" component of the timelike part of the field $\left(A^{l t}\right.$ parallel to $k$ in the "time" part of the space with no "space" components ) and by $A^{l s}$ the longitudinal component of the spacelike part. These components must satisfy $\Delta=0$ where $\Delta \equiv A^{l t}-A^{l s}$. The action of the gauge (equivalence) transformation is again of the form

$$
A^{\mu}(k) \longrightarrow A^{\mu}(k)+k^{\mu} \Lambda(k)
$$

where the Fourier transform is

$$
A^{\mu}(x) \longrightarrow A^{\mu}(x)+\partial^{\mu} \Lambda(x)
$$

and the Hilbert space has the same structure as in the $O(3,1)$ case. There is, of course, a problem with the quantum mechanical definition of the field for $q>1$ 
because the semisimple subgroup becomes non-compact and the finite dimensional representations are non-unitary. This problem will be studied elsewhere, using, for example, methods of the type discussed by Irim and Noz [3]. It is interesting to note that if the isometry group is $O(1,1)$, there is no little group (excluding the conformal symmetry for the massless particles) for massless and massive particles so there is no intrinsic differences between them from the point of view that we have discussed abore. In particular, there is no gauge symmetry (as for the Schwinger $\operatorname{model}[\tau])$.

\section{The Massless Spinor Field}

Consider now a massless spinor field in chiral representation in $3+1$ dimensions. The transformation property of a Wey spinor under the Lorentz group is given by

$$
\begin{gathered}
\phi_{L} \longrightarrow e^{\frac{1}{2} \sigma \cdot[\theta \hat{n}+i \zeta \dot{m}]} \phi_{L} \\
\phi_{R} \longrightarrow e^{\frac{1}{2} \sigma \cdot[\theta \hat{n}-i \varphi \hat{m}]} \phi_{R}
\end{gathered}
$$

where $\hat{m}$ is a unit 3 vector in the boost direction and $\hat{n}$ is the direction of the rotation axis. The parameters $\theta$ and $\varphi$ correspond, respectively to the rotation and the boost. The generators of the translation part of $E(2)$ (when the momentum $k$ is directed along the $\hat{\sim}$ axis) are $L_{1}$ and $L_{2}$ as defined above, represented by $\frac{\sigma^{1}}{2}$ and $\frac{\sigma^{2}}{2}$. The new spinors are, under the action of $L_{1}$,

$$
\begin{aligned}
\phi_{L}^{\prime}=e^{\frac{i}{2} \alpha\left(\sigma_{2}+i \sigma_{1}\right)} \dot{\phi}_{L} & =\left(1-\alpha\left(\begin{array}{ll}
0 & 0 \\
1 & 0
\end{array}\right)\right) \phi_{L} \\
- & 10-
\end{aligned}
$$




$$
\begin{gathered}
\Rightarrow\left(\begin{array}{c}
\dot{\phi}_{L}^{1} \\
\phi_{L}^{2}
\end{array}\right) \longrightarrow\left(\begin{array}{c}
\dot{\phi}_{L}^{1} \\
\dot{\phi}_{L}^{2}-\alpha \phi_{L}^{1}
\end{array}\right) \\
\dot{\phi}_{R}^{\prime}=\epsilon^{\frac{i}{3} \alpha\left(\sigma_{2}-i \sigma_{1}\right)} \phi_{R}=\left(1-\alpha\left(\begin{array}{ll}
0 & 1 \\
0 & 0
\end{array}\right)\right) \dot{\phi}_{R} \\
\Rightarrow\left(\begin{array}{c}
\phi_{R}^{1} \\
\dot{\phi}_{R}^{2}
\end{array}\right) \longrightarrow\left(\begin{array}{c}
\phi_{R}^{1}-\alpha \dot{\phi}_{R}^{2} \\
\dot{\phi}_{R}^{2}
\end{array}\right)
\end{gathered}
$$

and under $L_{2}$,

$$
\begin{gathered}
\dot{\phi}_{L}^{\prime}=e^{\frac{i}{2} \beta\left(i \sigma_{2}-i \sigma_{1}\right)} \phi_{L}=\left(1-i \beta\left(\begin{array}{ll}
0 & 0 \\
1 & 0
\end{array}\right)\right) \dot{\phi}_{L} \\
\Rightarrow\left(\begin{array}{c}
\dot{\phi}_{L}^{1} \\
\dot{\phi}_{L}^{2}
\end{array}\right) \longrightarrow\left(\begin{array}{c}
\dot{\phi}_{L}^{1} \\
\dot{\phi}_{L}^{2}-i \beta \phi_{L}^{1}
\end{array}\right) \\
\dot{\phi}_{R}^{\prime}=e^{\frac{i}{2} \beta\left(-i \sigma_{2}-\sigma_{1}\right)} \dot{\phi}_{R}=\left(1-i \beta\left(\begin{array}{ll}
0 & 1 \\
0 & 0
\end{array}\right)\right) \dot{\phi}_{R} \\
\Rightarrow\left(\begin{array}{c}
\phi_{R}^{1} \\
\dot{\phi}_{R}^{2}
\end{array}\right) \longrightarrow\left(\begin{array}{c}
\dot{\phi}_{R}^{1}-i \beta \dot{\phi}_{R}^{2} \\
\dot{\phi}_{R}^{2}
\end{array}\right)
\end{gathered}
$$

As we see, in this case, one can satisfy the triviality representation condition for the $E(2)$ translation part without equivalence relations; such a solution can be achieved by requiring the upper components of the left hand spinor, as well as the lower components of the right hand spinor to vanish identically. We know that in the chiral representation spatial inversion (the parity transformation) is represented by :

$$
\psi \rightarrow \gamma^{\circ} \dot{\psi}
$$

where $\psi$ is the 4 spinor $\left(\begin{array}{c}\dot{\phi}_{R} \\ \phi_{L}\end{array}\right)$ and $\psi^{\circ}$ is, in this representation, [8]

$$
\begin{aligned}
\gamma^{\circ}=\left(\begin{array}{cc}
0 & -I \\
-I & 0
\end{array}\right) & =\left(\begin{array}{cccc}
0 & 0 & -1 & 0 \\
0 & 0 & 0 & -1 \\
-1 & 0 & 0 & 0 \\
0 & -1 & 0 & 0
\end{array}\right) \\
& -11-
\end{aligned}
$$


Hence the parity transformation interchanges the upper components of the left handed and right handed spinors as well as the lower components. Such transformations mix physical components with identically zero components and therefore parity is violated. It is interesting to note that if we allow the freedom of spinor gauge then we can take the lower component of the right handed spinor and the upper component of the left handed spinor to be physical fields where the lower left and the upper right will be equivalent to themselves plus something. Parity is also riolated for this type of field.

\section{Massless, Gauge Invariant, Tensor Fields}

Let us consider now the case of a second rank massless tensor field $T^{\alpha 3}(k)$. We ask first if there is a massless tensor that is stable under the translation part of the little group without any equiralence relation, i.e., without gauge freedom. The strict stability condition is

$$
\tilde{\Lambda}_{t r} T \tilde{\Lambda}_{t r}^{T}=T
$$

where $\tilde{I}_{t r}$ belongs to the translation part of the little group. One finds that the most general tensor in the $O(3.1)$ geometry (when $k$ is in the $\hat{\tilde{z}}$ direction ) that satisfies this restriction can be written as a sum of three tensors, an antisymmetric part, a traceless part and a "conformal" part. The antisymmetric part,

$$
F^{\mu \nu}=\left(\begin{array}{cccc}
0 & \alpha(k) & \beta(k) & 0 \\
-\alpha(k) & 0 & 0 & -\alpha(k) \\
-\beta(k) & 0 & 0 & -\beta(k) \\
0 & \alpha(k) & \beta(k) & 0
\end{array}\right),
$$

is exactly of the form of the electromagnetic field tensor for propagating wares, where $\alpha(k)$ and $\beta(k)$ are the linear polarization components of the electric and 
magnetic fields. The traceless symmetric part,

$$
S^{\mu \nu}=\left(\begin{array}{cccc}
\gamma(k) & 0 & 0 & \gamma(k) \\
0 & 0 & 0 & 0 \\
0 & 0 & 0 & 0 \\
\gamma(k) & 0 & 0 & \gamma(k)
\end{array}\right)
$$

is a candidate to be the energy momentum tensor for a massless field with momentum $k$ and it is incleed of the form of the energy momentum tensor for the electromagnetic field or for gravitational radiation. What we have called the "conformal" part, has the form

$$
G^{\mu \nu}=\left(\begin{array}{cccc}
-\phi(k) & 0 & 0 & 0 \\
0 & \phi(k) & 0 & 0 \\
0 & 0 & \phi(k) & 0 \\
0 & 0 & 0 & \phi(k)
\end{array}\right)=\phi(k) \eta^{\mu \nu},
$$

where $\eta^{\mu \nu}$ is the flat metric $\operatorname{diag}(-,+,+,+)$, and $\phi(k)$ satisfies the massless condition. Fields of the form (25) and (26) are, in fact, necessarily massless if they are to maintain their form under Lorentz transformation. They are not in a definite representation of $O(3)$ but they are invariant in form under $E(2)$. On the other hand, a tensor of the form of $G^{\mu \nu}$ can also represent a massive field; a field with this structure is by definition a scalar under the action of the Lorentz group. If one takes $G^{\mu \nu}(x)$ to be a matter field that satisfies, in the massless case, $\partial^{\mu} \partial_{\mu} G^{\lambda \nu}(x)=0$, or in the massive case $\partial^{\mu} \partial_{\mu} G^{\lambda \nu}(x)=m^{2} G^{\lambda \nu}(x)$, it appears as (at least for free fields) essentially just one noninteracting massless (or massive ) INlein-Gordon field. One can, however, consider the conformal field as representation of the metric itself, i.e., as a general relativistic conformally flat metric. In the case of a conformally flat metric (e.g., of Robertson - Walker type [9]) of the form

$$
g^{\mu \nu}=\phi^{2}(x) \eta^{\mu \nu}
$$


the scalar curvature is [10]

$$
\begin{aligned}
R & =\phi^{-2}\left\{-2(d-1) \partial^{\mu} \partial_{\mu} \ln \phi(x)-(d-2)(d-1) \partial^{\mu} \ln \phi(x) \partial_{\mu} \ln \phi(x)\right\}= \\
& =\phi^{-2}\left\{-2(d-1) \frac{1}{\phi} \partial^{\mu} \partial_{\mu} \phi(x)+(d-1)(4-d) \frac{1}{\dot{\phi}^{2}} \partial^{\mu} \phi(x) \partial_{\mu} \phi(x)\right\}
\end{aligned}
$$

We know from the Einstein equations that if the trace of the energy - momentum tensor vanishes the scalar curvature $R$ also vanishes. In such a case, when $d=6$, we find from eq. (29) that $\partial^{\mu} \partial_{\mu} \phi^{2}(x)=0$, i.e., it must be a massless field.

\section{Gravitational Radiation and the Harmonic Coordinates Condi-} tion

We now turn to the four dimensional helicity 2 massless field. It is generally known that the four dimensional second rank tensor field contains states with helicity \pm 2 . On the other hand. the tensor fields we obtained in the previous section are of helicity zero type (the $S^{\mu \nu}$ and $G^{\mu \nu}$ ), and a helicity 1 field (the $F^{\mu \nu}$ ). We now wish to construct a second rank tensor with physical \pm 2 helicity polarization states Then the Hilbert space must be of a gauge type, i.e., it necessarily contains an equivalence relation.

We consider a symmetric, traceless second rank massless field in four dimensions. Such a tensor has in general 9 independent components of the form

$$
T^{\mu \nu}=\left(\begin{array}{cccc}
t^{00} & t^{01} & t^{02} & t^{03} \\
t^{01} & t^{11} & t^{12} & t^{13} \\
t^{02} & t^{12} & t^{22} & t^{23} \\
t^{03} & t^{13} & t^{23} & t^{33}
\end{array}\right)
$$

(where the traceless condition is assumed). The \pm 2 helicities are the fields,

$$
\begin{gathered}
e_{+}=t^{11}-t^{22}+2 i t^{12} \\
-14-
\end{gathered}
$$




$$
e_{-}=t^{11}-t^{22}-2 i t^{12} \text {. }
$$

With the definition.

$$
\tilde{\Lambda}_{t r} T \tilde{\Lambda}_{t r}^{T}=\tilde{T}^{\prime}
$$

one finds that the stability condition is,

$$
e_{+}^{\prime}=e_{+} e_{-}^{\prime}=e_{-}
$$

This implies the relations

$$
\begin{gathered}
t^{13}=t^{01} \\
t^{23}=t^{02} \\
t^{3.3}=2 t^{03}-t^{00},
\end{gathered}
$$

and the traceless condition becomes

$$
2\left(t^{00}-t^{03}\right)-\left(t^{11}+t^{22}\right)=0
$$

In addition to those identities, the fields $t^{00}, t^{03}, t^{01}$ and $t^{02}$ are non-physical fields and fall into an equivalence class defined by

$$
\begin{gathered}
t^{00} \sim t^{00}+\left(\alpha^{2}+\beta^{2}\right)\left(t^{00}-t^{03}\right)+2 \alpha t^{01}+2 \beta t^{02}+\alpha^{2} t^{11}+2 \alpha \beta t^{12}+\beta^{2} t^{22} \\
t^{03} \sim t^{03}+\left(\alpha^{2}+\beta^{2}\right)\left(t^{00}-t^{03}\right)+2 \alpha t^{01}+2 \beta t^{02}+\alpha^{2} t^{11}+2 \alpha \beta t^{12}+\beta^{2} t^{22} \\
t^{01} \sim t^{01}+\alpha\left(t^{00}-t^{03}\right)+\alpha t^{11}+\beta t^{12} \\
t^{02} \sim t^{02}+\beta\left(t^{00}-t^{03}\right)+\alpha t^{12}+\beta t^{22} \\
-15
\end{gathered}
$$


We see that the gauge freedom corresponding to this equivalence class can be written as

$$
T^{\mu \nu}(k) \longrightarrow T^{\mu \nu}(k)+k^{\mu} \Lambda^{\nu}(k)+k^{\nu} \Lambda^{\mu}(k)
$$

where $A^{\mu}(k)$ is defined by eqs. (36). One sees that $t^{00}-t^{03}$ is gauge invariant and can be a canclidate for a physical field, but the assumption of corariant commutation relations

$$
\left[a^{\mu \nu}(\mathbf{k}), a_{\sigma \lambda}^{\dagger}\left(b f k^{\prime}\right)\right]=\delta_{\sigma}^{\mu} \delta_{\lambda}^{\nu} \delta\left(\mathbf{k}-\mathbf{k}^{\prime}\right)
$$

(where the $a^{\mu \nu}$ 's are defined as the annihilation operators of the corresponding tensor field excitations) implies that $t^{00}-t^{03}$ is a zero norm (ghost) field. It follows that the condition that all the trace expectation values vanish is

$$
\left\langle\nu_{p h y s}^{\prime}\left|t^{11}+t^{22}\right| \nu_{p h y s}\right\rangle=0 .
$$

At this stage, with the help of (38) and a simple transformation of (30) to helicity diagonal form, one can see that there are only 2 physical components in the tensor field, i.e., $e_{+}$and $\epsilon_{-}$. The conditions (34) (satisfied as a Gupta-Bleuler type restriction on the Hilbert space) are exactly the conditions to eliminate negative norm states in this theory; as can be seen from the commutation relations. The gauge freedom now becomes,

$$
\begin{gathered}
t^{00} \sim t^{00}+\left(\alpha^{2}+\beta^{2}\right)\left(t^{00}-t^{03}\right)+2 \alpha t^{01}+2 \beta t^{02}+\left(\alpha^{2}-\beta^{2}\right) t^{11}+2 \alpha \beta t^{12} \\
t^{03} \sim t^{03}+\left(\alpha^{2}+\beta^{2}\right)\left(t^{00}-t^{03}\right)+2 \alpha t^{01}+2 \beta t^{02}+\left(\alpha^{2}-\beta^{2}\right) t^{11}+2 \alpha \beta t^{12} \\
t^{01} \sim t^{01}+\alpha\left(t^{00}-t^{03}\right)+\alpha t^{11}+\beta t^{12} \\
-16-
\end{gathered}
$$




$$
t^{02} \sim t^{02}+\beta\left(t^{00}-t^{03}\right)+\alpha t^{12}-\beta t^{11}
$$

The helicity zero states of this theory are of three types. The state corresponding to the components $t^{11}+t^{22}$ is a positive norm physical field eliminated from the Hilbert space by the traceless condition. The state corresponding to $t^{00}+t^{03}$ is a zero norm gauge equivalence class ghost. The field $t^{00}-t^{03}$ corresponds to a gauge invariant ghost. The helicity 1 fields of this theory, $t^{01} \pm i t^{02}$ are gauge equivalence class zero norm ghosts. All the gauge equivalence class ghosts can be taken to zero by an appropriate Lorentz transformation where the $t^{11}+t^{22}$ is eliminated in all Lorentz frames and $t^{00}-t^{03}$ is a gauge invariant ghost.

As we know, gravitational radiation is described by such a field. We then see that eqs. (34) and (3S) define harmonic coordinates, i.e., they satisfy the condition

$$
g^{\mu \nu} \Gamma_{\mu \nu}^{\lambda}=0
$$

known as the condition for harmonic coordinates [11].

\section{Summary}

In this paper we have studied the polarization properties of a massless local field in any dimension of spacetime. In the Fourier representation, the field is a finite dimensional irreducible representation of the corresponding $O(p . q)$ (or its universal covering) which leares a "light-like" vector invariant. From this group theoretical requirement, we are able to define the physical polarizations, and obtain the structure of the Hilbert space representing the states of such a field. We may further deduce that these fields must hare equivalence classes correspond to gauge symmetry: 
Acknowledgments One of us (L.P.H.) would like to thank Prof. B. Gruber for the opportunity to present an early form of this work at the symposium on Symmetries in Science VI, August 1992, in Bregenz, and to his colleagues there for discussions. He also wishes to thank Prof. S.L. Adler for his hospitality at the Institute for Adranced Study where this work was completed, and to the Monell Foundation for support during that period. 


\section{References}

1. E.P.Wigner, Ann Math. 40149 (1939).

2. R. Shaw, Nuovo Cim. 371086 (1965). See also R. Mirman, Nuec. Phys. B (Proc. Suppl.) $6110(1989)$.

3. Y.S.Kim and M.E.Noz, Theory and Applications of the Poincare Group, D.Reidel Comp., Dordrecht (1986).

4. S. Weinberg, Phys. Rev. 138B 988 (1965). See also, S. Weinberg, Phys. Rev. 134B SS2 (1964).

5. This extra term is generated systematically in the canonical quantization procedure of K. Haller, see e.g., S.P. Tomczak and K. Haller, Nuovo Cimento B 1 (1972). K. Haller and R.b. Sohn, Phys. Rev. A 20 (1979), K. Haller, Acta Phrs. Austriaca 42163 (1975); see also N. Shnerb and L.P. Horwitz, Phys. Rer. A, to be published, for the higher dimensional case.

6. A.O. Barut and R. Raczka, Theory of Group Representation and Application, p.200, PWN-Polish Scientific Publishers, Warsaw (197t).

7. N. Nakanishi and I. Ojima, Covariant Operator Formalisem of Gauge Field Theories and Quantum Gravity, World Scientific, Singapore (1991).

8. See e.g. , C. Itzytson and J.B. Zuber, Quantum Field Theory, McGraw-Hill, N.Y. (19S5).

9. F. Hoyle and J.V.Narliker, Action at a Distance in Physics and Cosmology , Freeman, Reading, (1974)

10. R.M.Wald, General Relativity, Univ. of Chicago Press, Chicago (1984).

11. See e.g., S. Weinberg, Gravitation and Cosmology, Wiley N.Y. (1978). 